\title{
Neutralization of CXCL12 attenuates established pulmonary hypertension in rats
}

Short Title: CXCL12 neutraligands in severe PH in the rat

Jennifer Bordenave ${ }^{1,2}$, Raphaël Thuillet ${ }^{1,2}$, Ly Tu $^{1,2}$, Carole Phan ${ }^{1,2}$, Amélie Cumont ${ }^{1,2}$, Claire Marsol $^{4}$, Alice Huertas ${ }^{1,2,3}$, Laurent Savale ${ }^{1,2,3}$, Marcel Hibert ${ }^{4}$, Jean-Luc Galzi ${ }^{4,5}$, Dominique Bonnet ${ }^{4}$, Marc Humbert ${ }^{1,2,3}$, Nelly Frossard ${ }^{4}$, Christophe Guignabert ${ }^{1,2}$

\footnotetext{
${ }^{1}$ INSERM UMR_S 999, Hôpital Marie Lannelongue, Le Plessis-Robinson, France;

${ }^{2}$ Université Paris-Sud and Université Paris-Saclay, Kremlin-Bicêtre, France;

${ }^{3}$ AP-HP, Service de Pneumologie, Centre de Référence de l'Hypertension Pulmonaire Sévère, DHU Thorax Innovation, Hôpital Bicêtre, France;

${ }^{4}$ Laboratoire d'Innovation Thérapeutique, UMR7200 CNRS/Université de Strasbourg, Labex MEDALIS, Faculté de Pharmacie, 74 route du Rhin, 67412 Illkirch;

${ }^{5}$ Biotechnologie et Signalisation Cellulaire, Ecole Supérieure de Biotechnologie de Strasbourg, UMR 7242 CNRS/Université de Strasbourg, Illkirch, France
}

\section{Address for Correspondence:}

Christophe Guignabert, Ph.D;

INSERM UMR_S 999;

Hôpital Marie Lannelongue;

133, Avenue de la Résistance;

92350 Le Plessis-Robinson, France.

Tel: +33-1-40948833; Fax: +33-1-40942522; christophe.guignabert@inserm.fr 


\section{Abstract}

Aims: The progressive accumulation of cells in pulmonary vascular walls is a key pathological feature of pulmonary arterial hypertension (PAH) that results in narrowing of the vessel lumen, but treatments targeting this mechanism are lacking. The $\mathrm{C}-\mathrm{X}-\mathrm{C}$ motif chemokine 12 (CXCL12) appears to be crucial in these processes. We investigated the activity of two CXCL12 neutraligands on experimental PH, using two complementary animal models.

\section{Graphical Abstract}
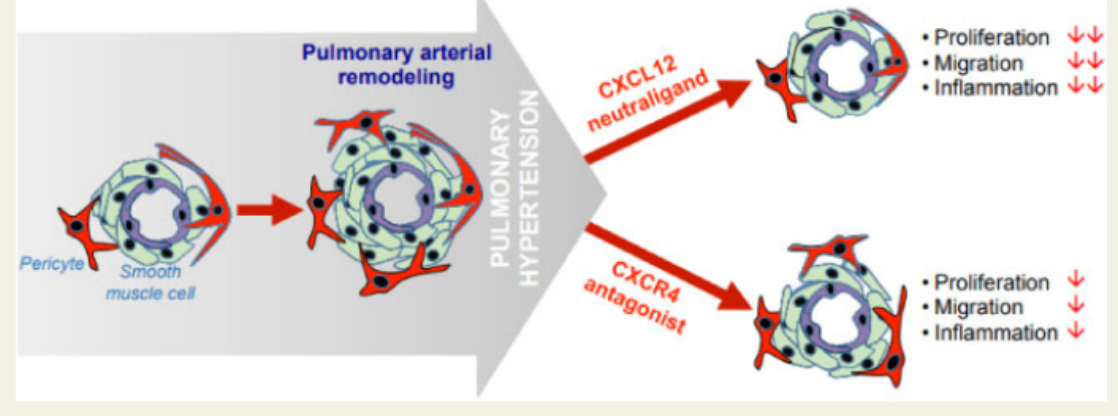

Methods and Results: Male Wistar rats were injected with monocrotaline or were subjected to SU5416 followed by 3-week Hypoxia to induce severe PH. After PH establishment, assessed by pulsed-wave Doppler echocardiography, MCT-injected or SuHx rats were randomized to receive CXCL12 neutraligands chalcone 4 or LIT-927 (100 mg/kg/day), the CXCR4 antagonist AMD3100 (5 mg/kg/day), or vehicle, for 2 or 3 weeks respectively. At the end of these treatment periods, echocardiographic and hemodynamic measurements were performed and tissue samples were collected for protein expression and histological analysis. Daily treatment of MCT-injected or SuHx rats with established PH with chalcone 4 or LIT-927 partially reversed established $\mathrm{PH}$, reducing total pulmonary vascular resistance, and remodelling of pulmonary arterioles. Consistent with these observations, we found that neutralization of CXCL12 attenuates right ventricular hypertrophy, pulmonary vascular remodelling, and decreases PA-SMC proliferation in lungs of MCT-injected rats and SuHx rats. Importantly, CXCL12 neutralization with either chalcone 4 or LIT-927 inhibited the migration of PA-SMCs and pericytes in vitro with a better efficacy than AMD3100. Finally, we found that CXCL12 neutralization decreases vascular pericyte coverage and macrophage infiltration in lungs of both MCT-injected and SuHx rats.

Conclusion: We report here a greater beneficial effect of CXCL12 neutralization versus the conventional CXCR4 blockade with AMD3100 in the MCT and SuHx rat models of severe $\mathrm{PH}$, supporting a role for CXCL12 in the progression of vascular complications in $\mathrm{PH}$ and opening to new therapeutic options. 
Keywords: Pulmonary arterial hypertension, vascular remodelling, animal model, therapeutic target, stromal cell-derived factor 1, chemokine 


\section{Abbreviations:}

3G5, 3G5-reactive ganglioside antigen

AU, arbitrary unit

CO, cardiac output

CXCL12, C-X-C motif chemokine 12

CXCR4, C-X-C motif chemokine receptor 4

CXCR7, C-X-C motif chemokine receptor 7

DAPI, 4',6-diamidino-2-phenylindole

ERK, extracellular signal-regulated kinase

$\mathbf{H} \& \mathbf{E}$, hematoxylin eosin

IL, interleukin

$\mathbf{L V}$, left ventricle

MCT, monocrotaline

mPAP, mean pulmonary arterial pressure

NG2, nerve/glial antigen 2

pAKT, phosphorylated protein kinase B

pERK, phosphorylated extracellular signal-regulated kinase

PAH, pulmonary arterial hypertension

PH, pulmonary hypertension

PVR, pulmonary vascular resistance

$\mathbf{R V}$, right ventricle

S, septum

SDF1, stromal cell-derived factor 1

SU or SU5416, 1,3-Dihydro-3-[(3,5-dimethyl-1H-pyrrol-2-yl)methylene]-2H-indol-2-one

SuHx, SU5416 plus chronic hypoxia

TPVR, total pulmonary vascular resistance

$\boldsymbol{\alpha}$-SMA, $\alpha$-smooth muscle actin 


\section{Introduction}

Pulmonary arterial hypertension (PAH) is a severe and incurable cardiopulmonary condition characterized by a marked and sustained increase in mean pulmonary artery pressure (mPAP) that ultimately leads to right ventricular failure and death ${ }^{1,2}$. Despite recent progresses, most patients still die from this life-threatening condition or fail to respond adequately to medical therapy with a 5 -year survival of $59 \%{ }^{3}$. Therefore, there is a substantial need to develop new therapies that target the cellular and molecular mechanisms that control the progressive obstructive pulmonary vascular remodelling in PAH. Emerging investigations emphasize the importance of recruitment and retention of inflammatory and vascular progenitor cells into the vessel wall and highlight roles for C-X-C motif chemokine 12 (CXCL12, also called stromal cell-derived factor-1) in the progression of experimental and human $\mathrm{PAH}{ }^{4-12}$.

CXCL12 is able to activate two chemokine receptors, CXCR4 and CXCR7, with different downstream signaling pathways. Besides this knowledge, the lung tissue distributions of these receptors, and their roles in PAH progression remain unknown ${ }^{13,14}$. To date, among the different types of nonpeptidic small molecules targeting the interaction of CXCL12 with CXCR4, cyclams and bicyclams such as AMD3100 are well-characterized agents ${ }^{15}$. However, the CXCR4 antagonist AMD3100 is also known to induce a rapid release of CXCL12 from bone marrow stroma to the circulation, leading to the release of stem cells into the circulation over their anchorage in bone marrow niches ${ }^{16}$. Because CXCR7, which binds to the ligand CXCL12 with higher affinity than CXCR4, also contributes to cell migration and homing induced by CXCL12, the notion that chemokine blockade could be an interesting strategy to be used as an alternative to conventional receptor blockade has emerged. In this context, we have recently identified chemokine-neutralizing molecules (neutraligands) that prevent CXCL12 from acting on its two main receptors CXCR4 and CXCR7, namely chalcone $4^{17,18}$ and the recently reported LIT-927, which is the first locally and orally active 
CXCL12 neutraligand with anti-inflammatory effect in a murine model of allergic airway hypereosinophilia ${ }^{19}$. However, the activity of CXCL12 neutraligands has not yet been studied in $\mathrm{PAH}$.

Therefore, in the present study, we explored the activities of chalcone 4 and LIT-927 on pulmonary hemodynamics and remodelling processes in cardiac tissues and pulmonary arteries in two complementary experimental models of severe pulmonary hypertension $(\mathrm{PH})$, namely the monocrotaline- and Sugen-hypoxia-induced models. In order to answer these questions, the CXCR4 antagonist AMD3100 was used as a control molecule in our in vivo and in vitro studies.

\section{Methods}

All animals were treated in accordance with the Guide for Care and Use of Laboratory Animals as adopted by our National Institute of Health and Medical Research (INSERM) and approval was granted by the Ethics Committee of the University Paris-Sud, Le PlessisRobinson, France ( $\underline{\left.n^{\circ} 01176.01\right)}$. In addition, all the experiments with human specimens were approved by the local ethics committee (Comite de Protection des Personnes [CPP] Ile-deFrance VII). All patients gave informed consent before the study. Detailed demographic and clinical characteristics are shown in Supplemental Fig. 1A.

\section{Animals and in vivo treatment}

Young male Wistar rats (100 g, Janvier Labs, Saint Berthevin, France) received a single subcutaneous injection of monocrotaline (MCT; $60 \mathrm{mg} / \mathrm{kg}$ ) or vehicle (Supplemental Fig. 1B). Male rats were used to minimize hormonal effects (e.g., of estrogen). At day-7, MCTinjected rats were randomly divided into four groups and treated for 2 weeks with daily 
intraperitoneal injections of vehicle [sodium carboxymethyl cellulose (CMC)], AMD3100 (5 $\mathrm{mg} / \mathrm{kg} /$ day $)$, chalcone 4 or LIT-927 (100 mg/kg/day) in CMC 17, 18, 20, 21. In parallel experiments, rats received a single subcutaneous injection of SU5416 (a VEGF-receptor antagonist; $20 \mathrm{mg} / \mathrm{kg}$ ) and were exposed to normobaric hypoxia for 3 weeks before return to room air (Supplemental Fig. 1C). Five weeks post SU5416 injection, pulsed-wave doppler during transthoracic echocardiography was used to evaluate pulmonary artery acceleration time (AT) to right ventricular ejection time (ET) ratio, using Vivid E9 (GE Healthcare, Velizy-Villacoublay, France). Then, the rats were randomized to receive vehicle (sodium carboxymethyl cellulose), AMD3100 (5 mg/kg/day, i.p.), chalcone 4 or LIT-927 (100 $\mathrm{mg} / \mathrm{kg} /$ day, i.p.) in $\mathrm{CMC}$ for 3 weeks ${ }^{22}$. At the end of these protocols, as previously described 23,24 , animals were anesthetized with isoflurane (2.0\% isoflurane in oxygen) was blindly assessed. A polyvinyl catheter was introduced into the right jugular vein and pushed through the right ventricle into the pulmonary artery to measure the mean pulmonary arterial pressure (mPAP). Cardiac output (CO) in rats was blindly measured using the thermodilution method. After measurement of hemodynamic parameters, the thorax was opened and the left lung immediately removed and frozen. The right lung was fixed in the distended state with formalin buffer. The right ventricular hypertrophy was assessed by the Fulton Index and the percentages of muscularized vessels were calculated.

\section{Isolation, culture, and treatment of Human PA-SMCs and pulmonary pericytes}

Primary human PA-SMCs and pericytes were isolated from human lung specimens. For PASMCs isolation, small pieces of freshly isolated arteries were cultured in DMEM media supplemented with $15 \%$ of foetal calf serum (FCS), 2 mM L-glutamine and antibiotics ${ }^{24-26}$. The isolated pulmonary PA-SMCs were strongly positive for alpha-smooth muscle actin ( $\alpha$ SMA), smooth muscle-specific SM22 protein and calponin, and negative for von Willebrand 
factor and CD31. Human pulmonary pericytes were isolated using an anti-3G5 antibody, prepared from murine hybridoma (ATCC, Molsheim, France), and anti-IgM magnetic beads (Dynal, Life Technologies, Saint-Aubin, France) on lung tissue fragment digested by collagenase type I (Gibco, Life Technologies). Pulmonary pericytes were cultured in Pericyte medium (Clinisciences, Nanterre, France) and are strongly positive for NG2 and PDGF receptor $\beta$. PA-SMCs and pericytes were used at passage $<5$. PA-SMC migration was assessed using the in vitro wound-healing assay. Briefly, confluent monolayers of PA-SMCs were scratch wounded, and then incubated with serum (10\% FBS) for 24 hours in presence of either vehicle (DMSO), AMD3100, chalcone 4 or LIT-927 at the indicated concentrations. Pericyte migration was assessed using a modified Boyden chamber chemotaxis system as described previously ${ }^{25,27}$. Briefly, pulmonary pericytes were stimulated to migrate for 6 hours in response to complete medium in presence of vehicle (DMSO), AMD3100, chalcone 4 or LIT-927 at the indicated concentrations.

\section{Western blot and Immunostaining}

Cells/tissues were homogenized and sonicated in RIPA buffer containing protease and phosphatase inhibitors and $30 \mu \mathrm{g}$ of protein was used to detect CXCL12, CXCR4, CXCR7, NG2, GAPDH and $\beta$-actin ${ }^{24-27}$ (Supplemental Table 1). Both CXCR4 and CXCR7 antibodies have been validated using siRNA knockdown assays (Supplemental Fig. 1D). Immunohistochemistry and immunofluorescent staining for CXCL12, CXCR4, CXCR7, proliferating cell nuclear antigen (PCNA), CD68, 3 G5 ganglioside, $\alpha$-SMA, and SM22 were performed in human and rat lung paraffin sections ${ }^{24-27}$ (Supplemental Table 1). Briefly, lung sections were deparaffinised and stained with hematoxylin and eosin (Sigma-Aldrich, SaintQuentin Fallavier, France), Picrosirius red, or incubated with the retrieval buffer. Then, sections were saturated with blocking buffer and incubated overnight with specific antibodies, followed by addition of the corresponding secondary fluorescent-labelled antibodies (Thermo 
Fisher Scientific, Saint-Aubin, France). Nuclei were labelled using DAPI (Thermo Fisher Scientific). Mounting was performed using ProLong Gold antifade reagent (Thermo Fisher Scientific). Images were taken using a LSM700 confocal microscope (Zeiss, Marly-le-Roi, France). Other lung sections were used for immunochemistry using vectastain $A B C$ kit according to the manufacturer's instructions (Abcys, Courtaboeuf, France) and counterstained with hematoxylin (Sigma-Aldrich). Images were taken using Eclipse 80i microscope (Nikon Instruments, Champigny-sur-Marne, France).

\section{Statistical analyses}

Statistically significant difference between results was tested using the nonparametric MannWhitney t-test or one-way ANOVA with Tukey post hoc tests. Significant difference was assumed at a $p$ value of $<0.05$. Continuous data are expressed as mean \pm SEM of at least three independent experiments or performed in triplicate or quintuplicate for technical replicates. $\mathrm{P}$ value $<0.05$ was considered statistically significant. Analyses were performed using GraphPad Prism v5.0 (La Jolla, CA, USA).

\section{Results}

CXCL12, CXCR4 and CXCR7 protein levels are increased in lungs from patients with idiopathic PAH (iPAH) and in lungs of rats with established PH

Immunofluorescence confocal microscopy in combination with Western blotting was first used to study the CXCL12, CXCR4, and CXCR7 tissue distribution in lung specimens from controls and iPAH patients (Figure 1). Consistent with previously published studies, our results indicate a 3.5- to 4-fold increase in CXCL12 and CXCR4 protein levels in lung 
specimens from iPAH patients; in contrast, both signals were weak in vessels from controls (Figure 1A-B). Whereas CXCR4 immunostaining was predominantly localized within walls of remodelled vessels, CXCL12 immunoreactivity was noted in the dysfunctional pulmonary endothelium and alveolar macrophages (Figure 1A-B). Our findings also reveal a 1.5-fold increase in CXCR7 protein levels in lung homogenates that concerns all layers of the remodelled arterial walls of iPAH patients, contrasting with its low immunoreactivity in control lung specimens (Figure 1C).

The two most widely used and complementary animal models of $\mathrm{PH}$ were next used to examine the lung protein expression patterns of CXCL12, CXCR4, and CXCR7. Consistent with our findings obtained with human specimens, we show here that CXCL12 (Figure 2A), and its two cognate receptors (Figure 2B-C) were substantially increased in lungs from rats with established PH induced by a single subcutaneous injection of either MCT or of SU5416 followed by chronic hypoxia when compared with control rat lungs.

Chronic treatment with AMD3100, chalcone 4, or LIT-927 attenuates established PH in MCT-injected rats

To determine the effect of treatments with either the CXCR4 antagonist AMD3100 or each of the CXCL12 neutraligands against the progression of established PH in MCT-injected rats, pulmonary hemodynamics and structural changes in the pulmonary arteries and cardiac tissues were studied (Figure 3 and Supplemental Fig. 1B and 2A). Serum levels of circulating CXCL12 were increased in MCT-injected rats, a phenomenon totally abolished with chronic treatments with the neutraligands chalcone 4 or LIT-927 versus vehicle (Supplemental Fig. 2B).

Twenty-one days post-MCT injection, a marked increase in values of mPAP, total pulmonary vascular resistance (TPVR) (Figure 3A), and in $\mathrm{RV} /(\mathrm{LV}+\mathrm{S})$ ratio (Figure 3B) were found in 
MCT-injected rats treated with vehicle when compared with control rats. Although no changes were found with AMD3100 treatment, MCT-injected rats treated with chalcone 4 or LIT-927 exhibited reduced mPAP associated with reduced TPVR elevation when compared with MCT-injected rats treated with vehicle (Figure 3A). In contrast to MCT-injected rats treated with AMD3100 or with chalcone 4, a substantial decrease in values of Fulton index (assessing right ventricular hypertrophy) was found in MCT-injected rats treated with LIT927 when compared with vehicle (Figure 3B). Of note, no differences were found in values of systemic pressures (mean systemic blood pressure: $90.7 \pm 5.5,90.4 \pm 11.8,87.8 \pm 6.7$, $87.5 \pm 13.9,80.8 \pm 14.4$, respectively, NS) between control rats and MCT-injected rats treated with vehicle, AMD3100, chalcone 4, or LIT-927.

Consistent with these results, the percentage of muscularized distal pulmonary arteries (Figure 3C) that was increased 8-fold in MCT-injected rats treated with vehicle when compared with control rats, was substantially decreased in MCT-injected rats treated with chalcone 4 or LIT-927 (Figure 3C). Furthermore, we also noted a reduced increase in the number of $\mathrm{PCNA}^{+}$cells per lung vessel in MCT-injected rats treated with AMD3100, chalcone 4, or LIT-927 when compared with vehicle (Figure 3C). In addition, we found a reduced collagen deposition in the right ventricle of MCT-injected rats treated with chalcone 4, or LIT-927 as compared with MCT-injected rats treated with vehicle, but not in MCT-injected rats treated with AMD3100 (Figure 3D).

Chronic treatment with AMD3100, chalcone 4, or LIT-927 attenuates established PH in

\section{SuHx rats}

To validate our findings in the MCT rat model, a 3-week daily treatment of SuHx rats with AMD3100, chalcone 4 or LIT-927 started 5 weeks post-SU5416 injection was next performed (Figure 4 and Supplemental Fig. 1C). Consistent with the MCT rat model, a substantial increase in the circulating levels of CXCL12 was found in the SuHx rat model of severe PH, 
a phenomenon totally abolished with chronic treatment of SuHx rats with either of the neutraligands as compared with vehicle (Supplemental Fig. 2C).

Eight weeks post-SU5416 injection, SuHx rats developed more severe experimental PH versus MCT-injected rats, as reflected by a marked increase in MPAP and in the ratio of acceleration time (AT) to ejection time (ET) (Supplemental Fig. 2D), and a decrease in cardiac output (CO) (Figure 4A). Although no significant changes were found in mPAP values in this severe model, SuHx rats treated with AMD3100, chalcone 4 or LIT-927 exhibited reduced TPVR elevation when compared to SuHx rat-treated with vehicle (Figure 4A). Furthermore, right ventricular hypertrophy, pulmonary vascular remodelling, and RV fibrosis were also more prominent in the SuHx versus MCT model (Figure 3 and 4, panels B-D).

Interestingly, in contrast to the AMD3100-treated MCT rats, AMD3100-treated SuHx rats exhibited reduced TPVR elevation and right ventricular hypertrophy as compared with SuHx rats treated with vehicle (Figure 3 and 4, panels A-B). Of note, no differences were also found in values systemic pressures (mean systemic blood pressure: $94.4 \pm 5.7,100.8 \pm 5.0$, $102.7 \pm 14.0,100.3 \pm 10.9,103.2 \pm 5.9$, respectively, NS) between controls and SuHx rats treated with vehicle, AMD3100, chalcone 4, or LIT-927.

In addition, our data also indicate that the pulmonary arterial muscularization, and the number of PCNA ${ }^{+}$cells per vessel were substantially decreased in SuHx rats treated with AMD3100, chalcone 4 or LIT-927 when compared with vehicle (Figure 4C-D and Supplemental Fig.

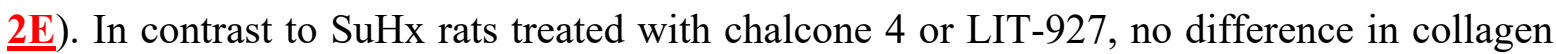
deposition in the right ventricle was observed in SuHx rats treated with AMD3100 (Figure 4D)

\section{CXCL12 neutraligands inhibit the migration of PA-SMCs and pericytes in vitro}


The CXCL12/CXCR4/CXCR7 axis directly contributes to cell migration, a phenomenon that plays a key role in the muscularization of distal pulmonary arteries in $\mathrm{PAH}^{9,25}$. First, we have validated that cultured human PA-SMCs and pulmonary pericytes express the CXCR4 and CXCR7 receptors (Supplement Fig. 3A) and that chalcone 4 and LIT-927 attenuate the CXCL12-induced phosphorylation of ERK and AKT (Supplement Fig. 3B-D). We therefore studied the effects of AMD3100, chalcone 4 and LIT-927 in vitro on migration of human PASMCs and pulmonary pericytes in primary cultures (Figure 5). In contrast to AMD3100, CXCL12 neutralization with chalcone 4 or LIT-927 inhibits the migration of cultured human PA-SMCs in a concentration-dependent manner (Figure 5A). Consistent with these findings, human pulmonary pericytes also exhibited decreased migration when exposed to chalcone 4 or LIT-927, with no effect of AMD3100 (Figure 5B).

\section{CXCL12 neutraligands decrease pericyte coverage of vessels and macrophage infiltration}

\section{in lungs from MCT-injected and SuHx rats}

To validate our in vitro observations, we then localized and quantified pericytes in lungs of MCT-injected and SuHx rats treated either with vehicle, AMD3100, chalcone 4, or LIT-927 (Figure 6). Western blotting and immunohistological studies revealed a substantial reduction in pericyte numbers around remodelled vessels in lungs of MCT and SuHx rats treated or not with chalcone 4, or LIT-927 when compared with lungs of MCT-injected and SuHx rats treated with vehicle (Figure 6A-B).

Because CXCL12 is well established to be responsible for recruiting macrophages $\stackrel{28,29}{\text {, we }}$ finally evaluated the effect of chronic treatments with AMD3100 and the neutraligands on macrophage infiltration in lungs of controls and treated rats. Our data indicate a substantial decrease in CD68 positive cells in both MCT-injected and SuHx rats treated with chalcone 4 and LIT-927 when compared with vehicle (Figure 6C-D). Furthermore, chronic treatment 
with AMD3100 decreased the number of CD68 positive cells only in the SuHx rats (Figure 6D).

\section{Discussion}

Pulmonary arterial hypertension is well characterized by progressive narrowing and obliteration of the pulmonary vasculature due to intrinsic proliferative potential and accumulation of resident pulmonary vascular cells and inflammatory cells in the vascular wall. However, the currently available drugs do not target these processes. Here, we report that neutralization of CXCL12 with small chemical compounds, the neutraligands chalcone 4 and LIT-927, improved pulmonary hemodynamics as well as lung and cardiac structure in rats with established $\mathrm{PH}$ in two complementary and well-established models of severe $\mathrm{PH}$. In addition, we obtained in vivo and in vitro evidence supporting the notion that the neutralization of CXCL12 is more effective than the conventional CXCR4 blockade with AMD3100.

This present translational research investigated the activity of two CXCL12 neutraligands against established $\mathrm{PH}$ in two complementary animal models of severe $\mathrm{PH}^{18-20}$. Hence, we firstly conducted immunofluorescence and confocal analyses of CXCL12, CXCR4, and CXCR7 protein levels in lungs from patients with iPAH and from rats with established $\mathrm{PH}$. Interestingly, we not only confirmed a more intense immunoreactivity for CXCL12 and $\underline{\text { CXCR4 in lungs from patients with iPAH }}{ }^{12}$ and from rodents with established $\mathrm{PH}^{4,6,21,30 \text {, }}$ 31 , but also highlighted an overexpression of CXCR7. Notably, we noted that the dysfunctional pulmonary endothelium and the alveolar macrophages are two local sources of CXCL12 in lungs of PAH patients and that PA-SMCs and several cells in the adventitia overexpress CXCR4 and CXCR7. We thus evaluated the effect of CXCL12 neutralization in MCT-injected and SuHx rats against established PH and found that daily treatments with 
either chalcone 4 or LIT-927, more effectively than AMD3100, attenuate pulmonary hemodynamics and lung vascular remodelling. Of interest to note is that the efficacy of AMD3100, chalcone-4, and LIT-927 treatments against established PH is more or less pronounced depending the animal models, perhaps because of the difference in the rapidity of disease progression or in the pathogenic mechanisms involved and their dynamic.

Herein, our present study supports that CXCL12 neutralization has better beneficial effect than the conventional CXCR4 blockade with AMD3100 in both MCT and SuHx rat models of severe $\mathrm{PH}$ that are characterized by a progressive and irreversible pulmonary vascular remodeling. Even if these two animal models do not reproduce the full spectrum of changes seen in lung specimens from iPAH patients, they are very valuable to validate new targets and/or treatments and to give insights into the disease mechanisms ${ }^{32}$. The current results are in line with the results obtained by our group and others showing that combined antagonism of CXCR4 and CXCR7 attenuates the chronic-hypoxia-induced $\mathrm{PH}^{6,30,31}$, and also with the results of Farkas et al. showing that CXCR4 inhibition with AMD3100 is able to prevent the increased muscularization and partially the obliteration of pulmonary arteries in the SuHx model ${ }^{21}$. Consistent with these in vivo observations, our in vitro findings obtained with primary cultures of human PA-SMCs and pulmonary pericytes indicate that CXCL12 neutralization, in contrast to CXCR4 antagonism with AMD3100, substantially inhibits cell migration in a concentration-dependent manner. Because accumulation of both PA-SMCs, pulmonary pericytes and inflammatory cells in walls of distal pulmonary arteries increases substantially during disease progression in $\mathrm{PAH}^{7,9,24,25,33}$, potent and selective inhibitors of the CXCL12/CXCR4/CXCR7 axis could open new therapeutic options in PAH, especially neutraligands which prevent binding of CXCL12 to the receptors CXCR4 and CXCR7 19, 20 . These neutraligands are, indeed, small selective molecules that can rapidly neutralize CXCL12 by direct binding and thus prevent CXCL12 from activating its CXCR4 and CXCR7 
receptors ${ }^{18-20}$. In addition to reduce migration of human PA-SMCs and pulmonary pericytes, chalcone 4 and LIT-927 attenuate the proliferation of human PA-SMCs in vitro (Supplemental Fig. 3E) and reduce numbers of macrophages in lungs from MCT-injected and SuHx rats. Remarkably, macrophages have been implicated in the pathogenesis of PAH 34-37. This mode of action that allow the blockade of CXCL12 without any effect on the two receptors favors cell homeostasis and the fact that these molecules influence several mechanisms involved in PAH pathobiology are particularly promising in this context.

It is now well established that CXCL12 expression is upregulated by hypoxia, as a result of HIF-1 and HIF-2 binding to its promoter ${ }^{38,39}$. However, inflammatory mediators such as IL1 an IL-6 can also induce CXCL12 expression in a CCAAT/enhancer binding protein $b$ (c/EBPb)-dependent manner ${ }^{40}$. Remarkably, CXCL12 neutralization does not affect levels of CXCL11 or CCL2 in the serum of rats treated with chalcone-4, LIT927, but substantially reduce circulating levels of macrophage migration inhibitory factor (MIF) (Supplemental Table 2). In addition, the promoter region of $C X C L 12$ contains binding sites for several transcriptional factors, including among others: Sp1, AP1, PARP1 and NF- $\kappa B^{41}$. Further investigations are thus needed to better understand the molecular mechanisms underlying the CXCL12 overexpression in lungs of PAH.

Although the CXCL12/CXCR4 axis plays an important role in the onset and progression of the disease ${ }^{4-12}$, little is known about the role of CXCR7 in PAH. Interestingly, CXCR7 appears to be critical for both the cardiovascular system development and vascular homeostasis in animal models. Decreased CXCR7 expression in zebrafish embryos inhibits blood vessel formation ${ }^{42}$, and its loss in mice causes early postnatal mortality as a result of myocardial degeneration and heart vessel damage ${ }^{43}$. In parallel, CXCR7 is also known to play an integral role in tumor progression by inducing tumor cell proliferation, adhesion, invasion, as well as tube formation in vitro and promotes tumor growth in vivo ${ }^{44-47}$. Studying 
the role of CXCR7 in PAH was not in the scope of this study, but will deserve further investigations.

In conclusion, our data demonstrate that the CXCL12/CXCR4/CXCR7 axis plays a central role in PAH and that CXCL12 neutralization appears to be an interesting strategy to be used as an alternative to conventional CXCR4 or CXCR7 blockade. Moreover, we found that these beneficial effects of CXCL12 neutraligands were associated with decreased pericyte coverage of remodelled pulmonary arterioles and reduced macrophage infiltration in lungs of MCTinjected and SuHx rats. Finally, this study offers important physiopathological insight into the role of the CXCL12/CXCR4/CXCR7 pathway and may have important implications for human PAH. 


\section{Acknowledgments:}

The authors thank Valérie Domergue and her team from the animal facility of UMS IPSIT for their help with the animals. This research was supported by grants from the French National Institute for Health and Medical Research (INSERM), the Centre National de la Recherche Scientifique (CNRS), the University of Paris-Sud and the Université Paris-Saclay, the Université de Strasbourg, the Marie Lannelongue Hospital, the French National Agency for Research (ANR) grant no. ANR-16-CE170014 (TAMIRAH), the Fondation pour la Recherche Médicale (FRM) grant no. DEQ20150331712 (Equipe FRM 2015) and in part by the Département Hospitalo-Universitaire (DHU) Thorax Innovation (TORINO), the Assistance Publique-Hôpitaux de Paris (AP-HP), Service de Pneumologie, Centre de Référence de l'Hypertension Pulmonaire Sévère, the LABEX Medalis (grant no ANR-10-LABX0034), the LabEx LERMIT (grant no ANR-10-LABX-0033), the French PAH patient association (HTAP France) and the french Fonds de Dotation "Recherche en Santé Respiratoire" - (FRSR) Fondation du Souffle (FdS). This research was also supported in part by an investigator-sponsored study (ISS) grant from GlaxoSmithKline (GSK). J.B. is supported by the FRM and C.P. by the FRSRFdS.

\section{Conflicts of interest:}

C.G., M.Hi., JL.G., D.B., and N.F. are the inventors on patent EP 16305 908.2. J.B., R.T., L.T., C.P., A.C., C.M., and A.H. have no conflict of interest to disclose. M.Hu. reports grants, personal fees and non-financial support from Actelion, Bayer, GSK, Gilead, Pfizer. M.Hu. has served as a consultant for Actelion, Bayer, GSK, Novartis, and Pfizer. L.S. reports grants, personal fees and non-financial support from Actelion, Bayer, GSK, MSD.

\section{Authors' contributions:}

J.B., L.T., N.F., and C.G. participated in the research design, J.B., R.T., L.T., C.P., A.C., C.M., and C.G. conducted the experiments and performed the data analysis, and C.G. wrote the manuscript. C.M., D.B., and M.Hi designed and synthesized the chemicals. All authors reviewed and revised the final version and approved manuscript submission. 


\section{References:}

1. Galie N, Humbert M, Vachiery JL, Gibbs S, Lang I, Torbicki A, Simonneau G, Peacock A, Vonk Noordegraaf A, Beghetti M, Ghofrani A, Gomez Sanchez MA, Hansmann G, Klepetko W, Lancellotti P, Matucci M, McDonagh T, Pierard LA, Trindade PT, Zompatori M, Hoeper M. 2015 ESC/ERS Guidelines for the diagnosis and treatment of pulmonary hypertension: The Joint Task Force for the Diagnosis and Treatment of Pulmonary Hypertension of the European Society of Cardiology (ESC) and the European Respiratory Society (ERS): Endorsed by: Association for European Paediatric and Congenital Cardiology (AEPC), International Society for Heart and Lung Transplantation (ISHLT). The European respiratory journal 2015;46:903-975.

2. Simonneau G, Gatzoulis MA, Adatia I, Celermajer D, Denton C, Ghofrani A, Gomez Sanchez MA, Krishna Kumar R, Landzberg M, Machado RF, Olschewski H, Robbins IM, Souza R. Updated clinical classification of pulmonary hypertension. Journal of the American College of Cardiology 2013;62:D34-41.

3. Boucly A, Weatherald J, Savale L, Jais X, Cottin V, Prevot G, Picard F, de Groote P, Jevnikar M, Bergot E, Chaouat A, Chabanne C, Bourdin A, Parent F, Montani D, Simonneau G, Humbert M, Sitbon O. Risk assessment, prognosis and guideline implementation in pulmonary arterial hypertension. The European respiratory journal 2017;50.

4. Costello CM, McCullagh B, Howell K, Sands M, Belperio JA, Keane MP, Gaine S, McLoughlin P. A role for the CXCL12 receptor, CXCR7, in the pathogenesis of human pulmonary vascular disease. The European respiratory journal 2012;39:1415-1424.

5. Farha S, Asosingh K, Xu W, Sharp J, George D, Comhair S, Park M, Tang WH, Loyd JE, Theil K, Tubbs R, Hsi E, Lichtin A, Erzurum SC. Hypoxia-inducible factors in human pulmonary arterial hypertension: a link to the intrinsic myeloid abnormalities. Blood 2011;117:3485-3493.

6. Gambaryan N, Perros F, Montani D, Cohen-Kaminsky S, Mazmanian M, Renaud JF, Simonneau G, Lombet A, Humbert M. Targeting of c-kit+ haematopoietic progenitor cells prevents hypoxic pulmonary hypertension. The European respiratory journal 2011;37:13921399.

7. Huertas A, Perros F, Tu L, Cohen-Kaminsky S, Montani D, Dorfmuller P, Guignabert C, Humbert M. Immune dysregulation and endothelial dysfunction in pulmonary arterial hypertension: a complex interplay. Circulation 2014;129:1332-1340.

8. McCullagh BN, Costello CM, Li L, O'Connell C, Codd M, Lawrie A, Morton A, Kiely DG, Condliffe R, Elliot C, McLoughlin P, Gaine S. Elevated plasma CXCL12alpha is associated with a poorer prognosis in pulmonary arterial hypertension. PLoS One 2015;10:e0123709.

9. Ricard N, Tu L, Le Hiress M, Huertas A, Phan C, Thuillet R, Sattler C, Fadel E, Seferian A, Montani D, Dorfmuller P, Humbert M, Guignabert C. Increased pericyte coverage mediated by endothelial-derived fibroblast growth factor- 2 and interleukin- 6 is a source of smooth muscle-like cells in pulmonary hypertension. Circulation 2014;129:1586-1597.

10. Yang T, Li ZN, Chen G, Gu Q, Ni XH, Zhao ZH, Ye J, Meng XM, Liu ZH, Xiong CM, He JG. Increased levels of plasma CXC-Chemokine Ligand 10, 12 and 16 are associated with right ventricular function in patients with idiopathic pulmonary arterial hypertension. Heart Lung 2014;43:322-327.

11. Hashimoto-Kataoka T, Hosen N, Sonobe T, Arita Y, Yasui T, Masaki T, Minami M, Inagaki T, Miyagawa S, Sawa Y, Murakami M, Kumanogoh A, Yamauchi-Takihara K, Okumura M, Kishimoto T, Komuro I, Shirai M, Sakata Y, Nakaoka Y. Interleukin-6/interleukin-21 signaling axis is critical in the pathogenesis of pulmonary arterial hypertension. Proc Natl Acad Sci U S A 2015;112:E2677-2686. 
12. Montani D, Perros F, Gambaryan N, Girerd B, Dorfmuller P, Price LC, Huertas A, Hammad H, Lambrecht B, Simonneau G, Launay JM, Cohen-Kaminsky S, Humbert M. C-kit-positive cells accumulate in remodeled vessels of idiopathic pulmonary arterial hypertension. American journal of respiratory and critical care medicine 2011;184:116-123.

13. Balabanian K, Lagane B, Infantino S, Chow KY, Harriague J, Moepps B, Arenzana-Seisdedos $\mathrm{F}$, Thelen M, Bachelerie F. The chemokine SDF-1/CXCL12 binds to and signals through the orphan receptor $\mathrm{RDC1}$ in $\mathrm{T}$ lymphocytes. The Journal of biological chemistry 2005;280:35760-35766.

14. Liu L, Chen JX, Zhang XW, Sun Q, Yang L, Liu A, Hu S, Guo F, Liu S, Huang Y, Yang Y, Qiu HB. Chemokine receptor 7 overexpression promotes mesenchymal stem cell migration and proliferation via secreting Chemokine ligand 12. Sci Rep 2018;8:204.

15. De Clercq E. The AMD3100 story: the path to the discovery of a stem cell mobilizer (Mozobil). Biochem Pharmacol 2009;77:1655-1664.

16. Dar A, Schajnovitz A, Lapid K, Kalinkovich A, Itkin T, Ludin A, Kao WM, Battista M, Tesio M, Kollet O, Cohen NN, Margalit R, Buss EC, Baleux F, Oishi S, Fujii N, Larochelle A, Dunbar CE, Broxmeyer HE, Frenette PS, Lapidot T. Rapid mobilization of hematopoietic progenitors by AMD3100 and catecholamines is mediated by CXCR4-dependent SDF-1 release from bone marrow stromal cells. Leukemia 2011;25:1286-1296.

17. Daubeuf F, Hachet-Haas M, Gizzi P, Gasparik V, Bonnet D, Utard V, Hibert M, Frossard N, Galzi JL. An antedrug of the CXCL12 neutraligand blocks experimental allergic asthma without systemic effect in mice. The Journal of biological chemistry 2013;288:11865-11876.

18. Gasparik V, Daubeuf F, Hachet-Haas M, Rohmer F, Gizzi P, Haiech J, Galzi JL, Hibert M, Bonnet D, Frossard N. Prodrugs of a CXC Chemokine-12 (CXCL12) Neutraligand Prevent Inflammatory Reactions in an Asthma Model in Vivo. ACS Med Chem Lett 2012;3:10-14.

19. Regenass P, Abboud D, Daubeuf F, Lehalle C, Gizzi P, Riche S, Hachet-Haas M, Rohmer F, Gasparik V, Boeglin D, Haiech J, Knehans T, Rognan D, Heissler D, Marsol C, Villa P, Galzi JL, Hibert M, Frossard N, Bonnet D. Discovery of a Locally and Orally Active CXCL12 Neutraligand (LIT-927) with Anti-inflammatory Effect in a Murine Model of Allergic Airway Hypereosinophilia. J Med Chem 2018;61:7671-7686.

20. Hachet-Haas M, Balabanian K, Rohmer F, Pons F, Franchet C, Lecat S, Chow KY, Dagher R, Gizzi P, Didier B, Lagane B, Kellenberger E, Bonnet D, Baleux F, Haiech J, Parmentier M, Frossard N, Arenzana-Seisdedos F, Hibert M, Galzi JL. Small neutralizing molecules to inhibit actions of the chemokine CXCL12. The Journal of biological chemistry 2008;283:23189-23199.

21. Farkas D, Kraskauskas D, Drake JI, Alhussaini AA, Kraskauskiene V, Bogaard HJ, Cool CD, Voelkel NF, Farkas L. CXCR4 inhibition ameliorates severe obliterative pulmonary hypertension and accumulation of C-kit(+) cells in rats. PLoS One 2014;9:e89810.

22. Schaefer CJ, Ruhrmund DW, Pan L, Seiwert SD, Kossen K. Antifibrotic activities of pirfenidone in animal models. European respiratory review : an official journal of the European Respiratory Society 2011;20:85-97.

23. Guignabert C, Phan C, Seferian A, Huertas A, Tu L, Thuillet R, Sattler C, Le Hiress M, Tamura Y, Jutant EM, Chaumais MC, Bouchet S, Maneglier B, Molimard M, Rousselot P, Sitbon O, Simonneau G, Montani D, Humbert M. Dasatinib induces lung vascular toxicity and predisposes to pulmonary hypertension. J Clin Invest 2016;126:3207-3218.

24. Tamura Y, Phan C, Tu L, Le Hiress M, Thuillet R, Jutant EM, Fadel E, Savale L, Huertas A, Humbert M, Guignabert C. Ectopic upregulation of membrane-bound IL6R drives vascular remodeling in pulmonary arterial hypertension. J Clin Invest 2018;128:1956-1970.

25. Tu L, De Man FS, Girerd B, Huertas A, Chaumais MC, Lecerf F, Francois C, Perros F, Dorfmuller P, Fadel E, Montani D, Eddahibi S, Humbert M, Guignabert C. A critical role for 
p130Cas in the progression of pulmonary hypertension in humans and rodents. Am J Respir Crit Care Med 2012;186:666-676.

26. Huertas A, Tu L, Thuillet R, Le Hiress M, Phan C, Ricard N, Nadaud S, Fadel E, Humbert M, Guignabert C. Leptin signalling system as a target for pulmonary arterial hypertension therapy. Eur Respir J 2015;45:1066-1080.

27. Tu L, Dewachter L, Gore B, Fadel E, Dartevelle P, Simonneau G, Humbert M, Eddahibi S, Guignabert C. Autocrine fibroblast growth factor-2 signaling contributes to altered endothelial phenotype in pulmonary hypertension. Am J Respir Cell Mol Biol 2011;45:311-322.

28. Sanchez-Martin L, Estecha A, Samaniego R, Sanchez-Ramon S, Vega MA, Sanchez-Mateos P. The chemokine CXCL12 regulates monocyte-macrophage differentiation and RUNX3 expression. Blood 2011;117:88-97.

29. Li M, Ransohoff RM. The roles of chemokine CXCL12 in embryonic and brain tumor angiogenesis. Semin Cancer Biol 2009;19:111-115.

30. Sartina E, Suguihara C, Ramchandran S, Nwajei P, Rodriguez M, Torres E, Hehre D, Devia C, Walters MJ, Penfold ME, Young KC. Antagonism of CXCR7 attenuates chronic hypoxiainduced pulmonary hypertension. Pediatric research 2012;71:682-688.

31. Young KC, Torres E, Hatzistergos KE, Hehre D, Suguihara C, Hare JM. Inhibition of the SDF-1/CXCR4 axis attenuates neonatal hypoxia-induced pulmonary hypertension. Circulation research 2009;104:1293-1301.

32. Bonniaud P, Fabre A, Frossard N, Guignabert C, Inman M, Kuebler WM, Maes T, Shi W, Stampfli M, Uhlig S, White E, Witzenrath M, Bellaye PS, Crestani B, Eickelberg O, Fehrenbach H, Guenther A, Jenkins G, Joos G, Magnan A, Maitre B, Maus UA, Reinhold P, Vernooy JHJ, Richeldi L, Kolb M. Optimising experimental research in respiratory diseases: an ERS statement. The European respiratory journal 2018;51.

33. Rabinovitch M, Guignabert C, Humbert M, Nicolls MR. Inflammation and immunity in the pathogenesis of pulmonary arterial hypertension. Circ Res 2014;115:165-175.

34. Amsellem V, Abid S, Poupel L, Parpaleix A, Rodero M, Gary-Bobo G, Latiri M, DuboisRande JL, Lipskaia L, Combadiere C, Adnot S. Roles for the CX3CL1/CX3CR1 and CCL2/CCR2 Chemokine Systems in Hypoxic Pulmonary Hypertension. American journal of respiratory cell and molecular biology 2017;56:597-608.

35. Florentin J, Coppin E, Vasamsetti SB, Zhao J, Tai YY, Tang Y, Zhang Y, Watson A, Sembrat J, Rojas M, Vargas SO, Chan SY, Dutta P. Inflammatory Macrophage Expansion in Pulmonary Hypertension Depends upon Mobilization of Blood-Borne Monocytes. J Immunol 2018;200:3612-3625.

36. Kumar R, Mickael C, Kassa B, Gebreab L, Robinson JC, Koyanagi DE, Sanders L, Barthel L, Meadows C, Fox D, Irwin D, Li M, McKeon BA, Riddle S, Dale Brown R, Morgan LE, Evans CM, Hernandez-Saavedra D, Bandeira A, Maloney JP, Bull TM, Janssen WJ, Stenmark KR, Tuder RM, Graham BB. TGF-beta activation by bone marrow-derived thrombospondin1 causes Schistosoma- and hypoxia-induced pulmonary hypertension. Nat Commun 2017;8:15494.

37. Tian W, Jiang X, Tamosiuniene R, Sung YK, Qian J, Dhillon G, Gera L, Farkas L, Rabinovitch M, Zamanian RT, Inayathullah M, Fridlib M, Rajadas J, Peters-Golden M, Voelkel NF, Nicolls MR. Blocking macrophage leukotriene b4 prevents endothelial injury and reverses pulmonary hypertension. Sci Transl Med 2013;5:200ra117.

38. Hitchon C, Wong K, Ma G, Reed J, Lyttle D, El-Gabalawy H. Hypoxia-induced production of stromal cell-derived factor 1 (CXCL12) and vascular endothelial growth factor by synovial fibroblasts. Arthritis Rheum 2002;46:2587-2597. 
39. Martin SK, Diamond P, Williams SA, To LB, Peet DJ, Fujii N, Gronthos S, Harris AL, Zannettino AC. Hypoxia-inducible factor-2 is a novel regulator of aberrant CXCL12 expression in multiple myeloma plasma cells. Haematologica 2010;95:776-784.

40. Calonge E, Alonso-Lobo JM, Escandon C, Gonzalez N, Bermejo M, Santiago B, Mestre L, Pablos JL, Caruz A, Alcami J. c/EBPbeta is a major regulatory element driving transcriptional activation of the CXCL12 promoter. J Mol Biol 2010;396:463-472.

41. Garcia-Moruja C, Alonso-Lobo JM, Rueda P, Torres C, Gonzalez N, Bermejo M, Luque F, Arenzana-Seisdedos F, Alcami J, Caruz A. Functional characterization of SDF-1 proximal promoter. J Mol Biol 2005;348:43-62.

42. Perlin JR, Talbot WS. Signals on the move: chemokine receptors and organogenesis in zebrafish. Sci STKE 2007;2007:pe45.

43. Gerrits H, van Ingen Schenau DS, Bakker NE, van Disseldorp AJ, Strik A, Hermens LS, Koenen TB, Krajnc-Franken MA, Gossen JA. Early postnatal lethality and cardiovascular defects in CXCR7-deficient mice. Genesis 2008;46:235-245.

44. Hao M, Zheng J, Hou K, Wang J, Chen X, Lu X, Bo J, Xu C, Shen K, Wang J. Role of chemokine receptor CXCR7 in bladder cancer progression. Biochem Pharmacol 2012;84:204214.

45. Zhao Q, Zhang P, Qin G, Ren F, Zheng Y, Qiao Y, Sun T, Zhang Y. Role of CXCR7 as a Common Predictor for Prognosis in Solid Tumors: a Meta-Analysis. J Cancer 2018;9:31383148 .

46. Long P, Sun F, Ma Y, Huang Y. Inhibition of CXCR4 and CXCR7 for reduction of cell proliferation and invasion in human endometrial cancer. Tumour Biol 2016;37:7473-7480.

47. Miao Z, Luker KE, Summers BC, Berahovich R, Bhojani MS, Rehemtulla A, Kleer CG, Essner JJ, Nasevicius A, Luker GD, Howard MC, Schall TJ. CXCR7 (RDC1) promotes breast and lung tumor growth in vivo and is expressed on tumor-associated vasculature. Proc Natl Acad Sci U S A 2007;104:15735-15740. 


\section{Figure Legends:}

Figure 1: Increased expressions of CXCL12, CXCR4, and CXCR7 in lungs patients with idiopathic pulmonary arterial hypertension (iPAH). (A) Representative images of CXCL12 (red), (B) CXCR4 (red), and (C) CXCR7 (red) staining with SM22 (green) and DAPI (blue) and Western blots with quantification of the CXCL12:GAPDH, CXCR4:GAPDH, and CXCR7:GAPDH ratios in lungs from control subjects and iPAH. Comparisons were made using the nonparametric Mann-Whitney t-test. Scale bar $=50 \mu \mathrm{m}$ in all sections. Horizontal lines display the mean $\pm \operatorname{SEM}(n=5-7)$. ${ }^{*} p$-value $<0.05$, *** $p$-value $<0.001$ compared with control. AU = arbitrary unit; DAPI $=4$ ',6-diamidino-2-phenylindole.

A
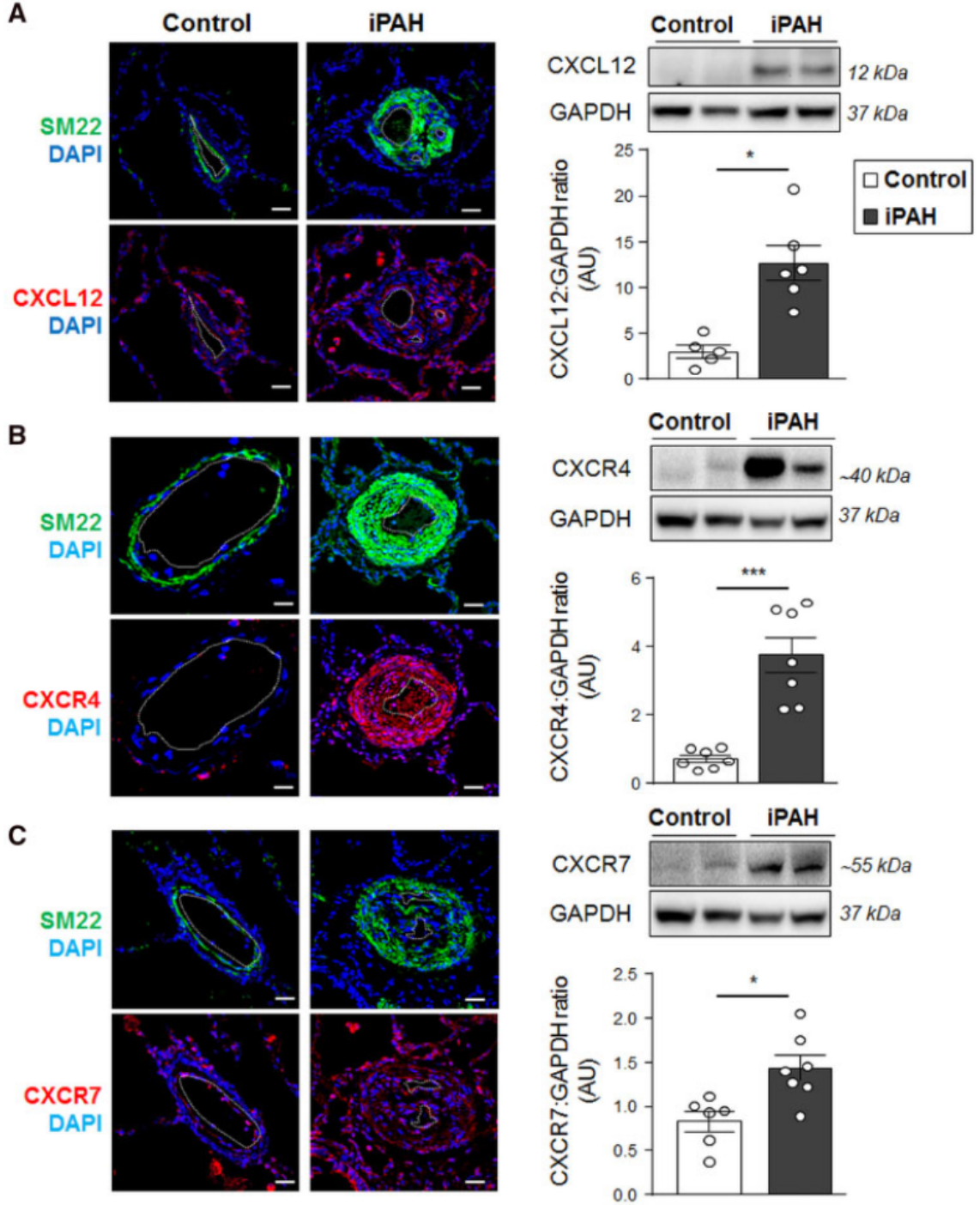

Figure 2: Increased expressions of CXCL12, CXCR4, and CXCR7 in lungs of monocrotaline (MCT)-injected and sugen/hypoxia (SuHx) rats with established pulmonary hypertension $(\mathrm{PH})$. (A) Representative images of CXCL12 (red), (B) CXCR4 (red), and (C) CXCR7 (red) staining with asmooth muscle actin ( $\alpha-S M A ;$ green) and DAPI (blue) and Western blots with quantification of the CXCL12:GAPDH, CXCR4:GAPDH, and CXCR7:GAPDH ratios in lungs from control, MCT-injected and SuHx rats (at Week-3 and Week-5, respectively). Scale bar $=50 \mu \mathrm{m}$ in all sections. Horizontal lines display the mean \pm SEM $(n=5-7)$. Comparisons were made using the nonparametric MannWhitney t-test. * p-value $<0.05$, ** $p$-value $<0.01$, ${ }^{* * *} p$-value $<0.001$ compared with control. AU = arbitrary unit; DAPI = 4',6-diamidino-2-phenylindole. 
A
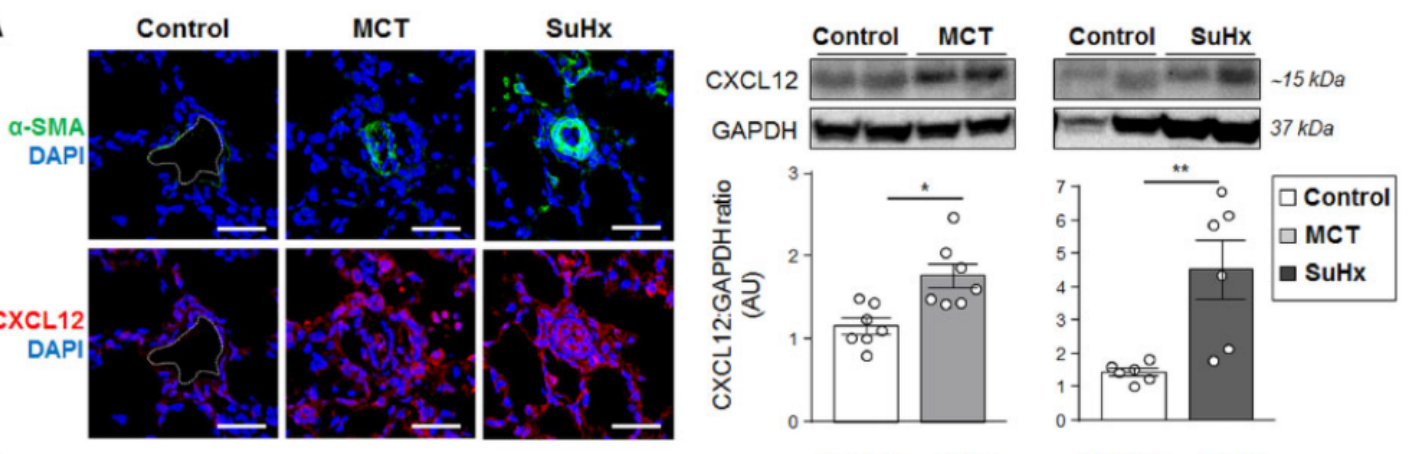

B
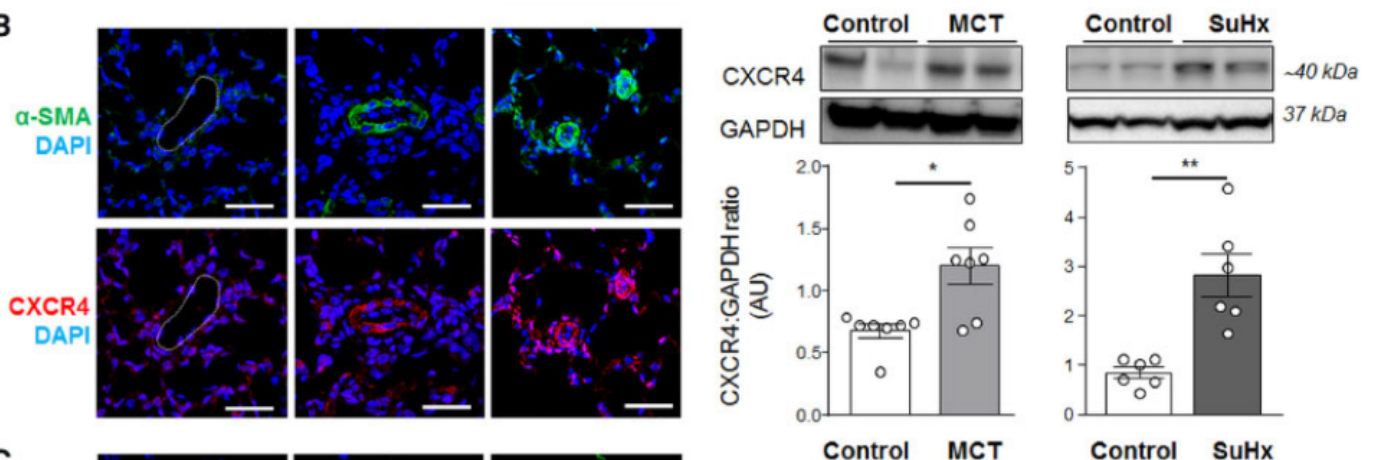

C
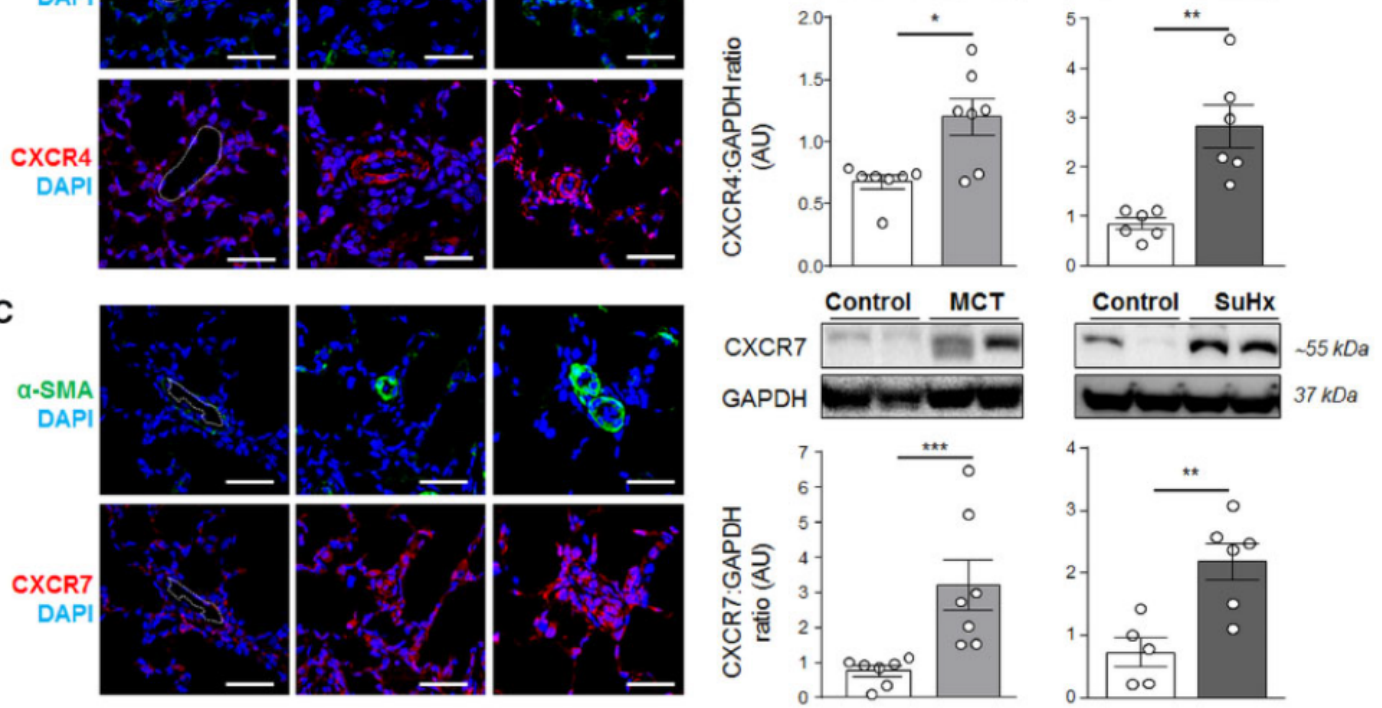

Figure 3: Efficacy of AMD3100, chalcone 4, and LIT-927 in the monocrotaline (MCT) rat model of severe PH. (A) Values of mean pulmonary arterial pressure (mPAP), cardiac output (CO), total pulmonary vascular resistance (TPVR), and of (B) Fulton index in vehicle-, AMD3100-, chalcone 4-, and LIT-927 treated rats. (C) Representative images of haematoxylin and eosin (H\&E)-, $\alpha$-smooth muscle (SM) actin- and proliferating cell nuclear antigen (PCNA)-stained sections of distal pulmonary arteries and quantification of the percentage of muscularized distal pulmonary arteries and of the number of PCNA positive cells per vessel in lungs of vehicle-, AMD3100-, chalcone 4-, and LIT-927 treated rats. (D) Representative images of picrosirius staining of tissue section of right ventricle myocardium of control and monocrotaline (MCT)-injected rats treated with vehicle- AMD3100-, chalcone 4-, and LIT-927. Horizontal lines display the mean \pm SEM $(n=4-5)$. Comparisons were made using 1-way ANOVA with Tukey's post hoc tests. ${ }^{*} p$-value $<0.05$; ${ }^{* *} p$-value $<0.01$; ${ }^{* * *} p$-value $<$ 0.001 ; ${ }^{* * * *} p$-value $<0.0001$ versus control rats. \# p-value $<0.05$; \#\# p-value $<0.01$; \#\# p-value $<$ 0.001 ; \#\# p-value < 0.0001 versus vehicle treated rats exposed to MCT. $\$ p$-value $<0.05 ; \$ \$ p$ value $<0.01 ; \$ \$ \$ p$-value $<0.001$; versus AMD3100-treated rats exposed to MCT Scale bar $=50 \mu \mathrm{m}$ in all sections. $A U$ = arbitrary unit; $L V=$ left ventricle; $R V=$ right ventricle; $S=$ septum. 

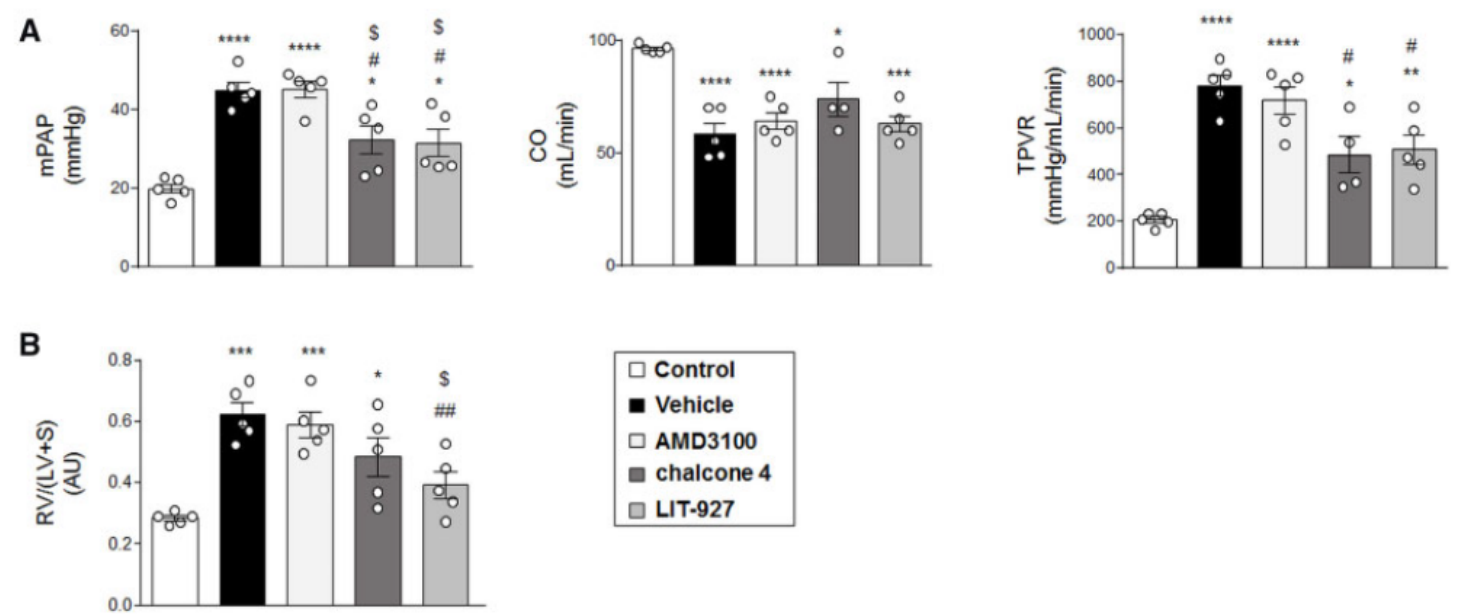

C
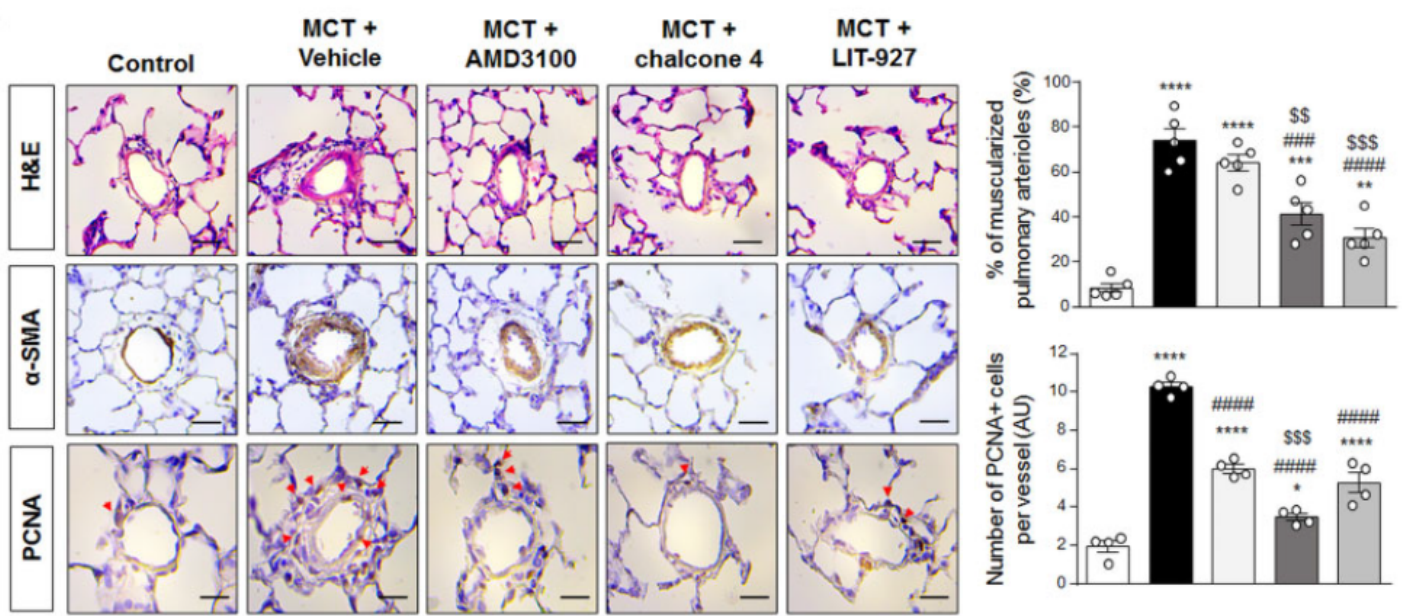

D
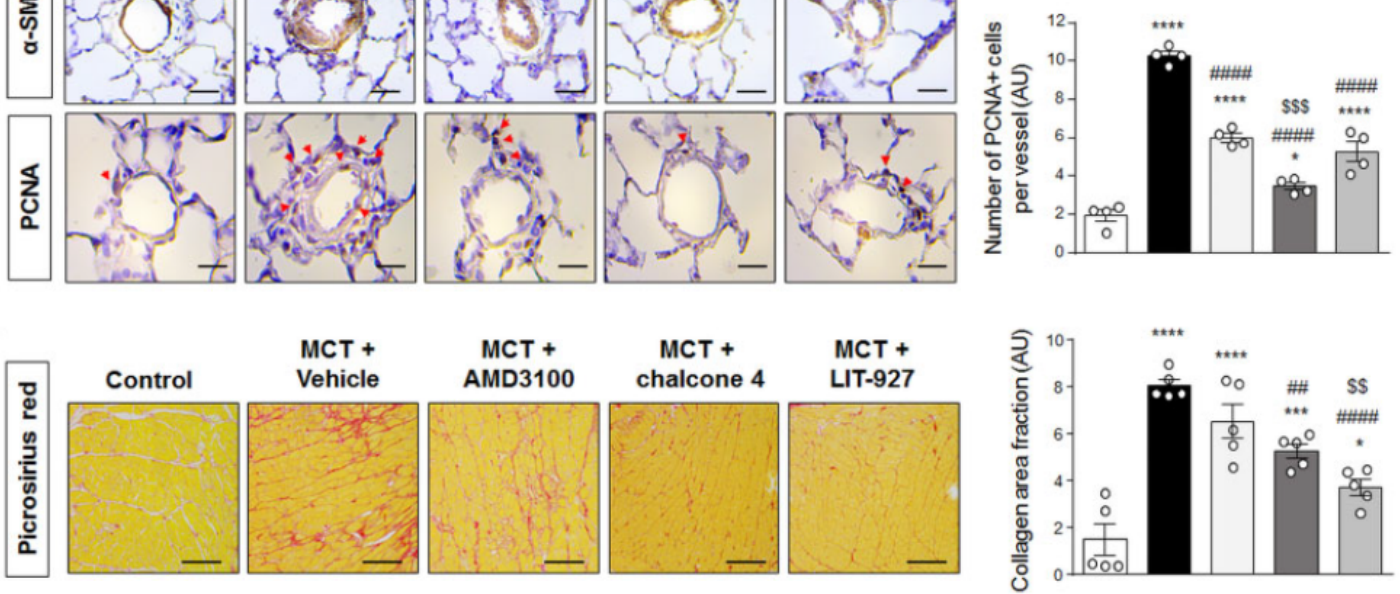

Figure 4: Efficacy of AMD3100, chalcone 4, and LIT-927 in the Sugen-hypoxia (SuHx) rat model of severe PH. (A) Values of mean pulmonary arterial pressure (mPAP), cardiac output (CO), total pulmonary vascular resistance (TPVR), and of (B) Fulton index in vehicle-, AMD3100-, chalcone 4-, and LIT-927 treated SuHx rats. (C) Representative images of haematoxylin and eosin (H\&E)-, $\alpha-$ smooth muscle (SM) actin- and proliferating cell nuclear antigen (PCNA)-stained sections of distal pulmonary arteries and quantification of the percentage of muscularized distal pulmonary arteries and of the number of PCNA positive cells per vessel in lungs of vehicle-, AMD3100-, chalcone 4-, and LIT927 treated SuHx rats. (D) Representative images of picrosirius staining of tissue section of right ventricle myocardium of control and SuHx rats treated with vehicle- AMD3100-, chalcone 4-, and LIT927. Horizontal lines display the mean $\pm \operatorname{SEM}(n=4)$. Comparisons were made using 1-way ANOVA with Tukey's post hoc tests. ${ }^{* * *} p$-value $<0.001 ;{ }^{* * *} p$-value $<0.0001$ versus control rats. \# $p$-value $<$ 0.05 ; \#\# p-value $<0.01$; \#\#\# p-value < 0.001; \#\#\# p-value $<0.0001$ versus vehicle treated SuHx rats. $\$ p$-value < 0.05; $\$ \$ p$-value $<0.01 ; \$ \$ \$ p$-value $<0.001 ; \$ \$ \$ p$-value $<0.0001$ versus AMD3100treated SuHx rats. Scale bar $=50 \mu \mathrm{m}$ in all sections. $\mathrm{AU}=$ arbitrary unit; LV = left ventricle; RV = right ventricle; $\mathrm{S}=$ septum. 
A
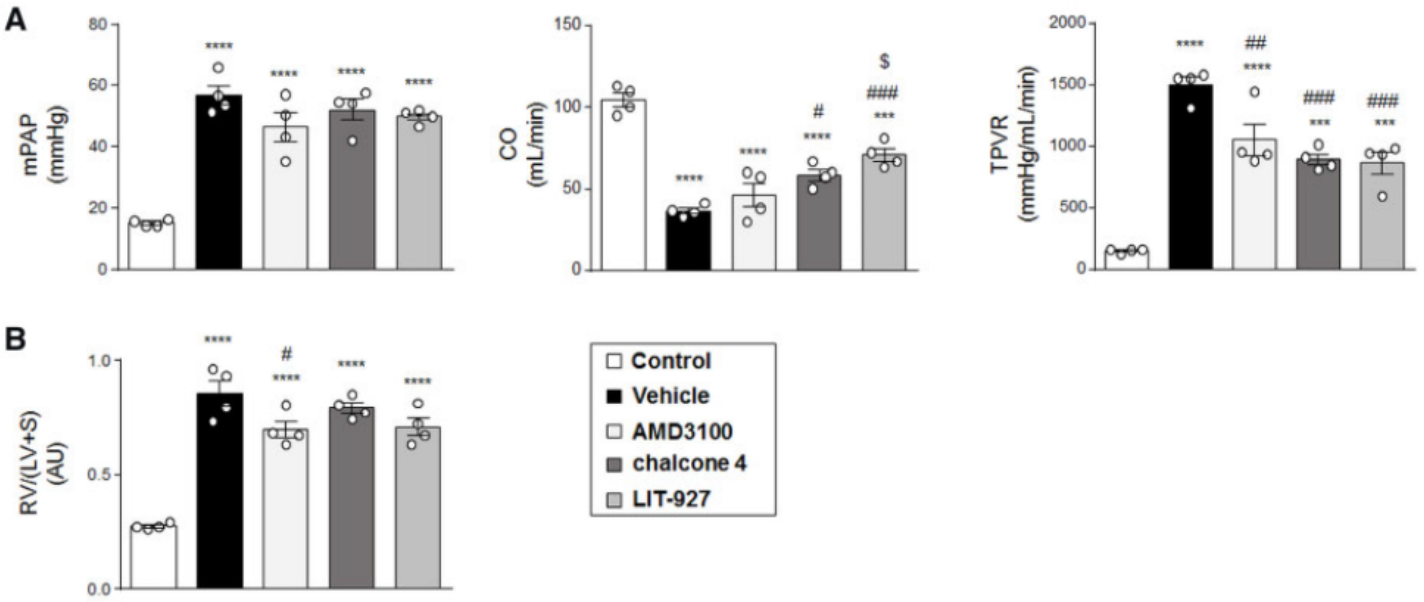

C

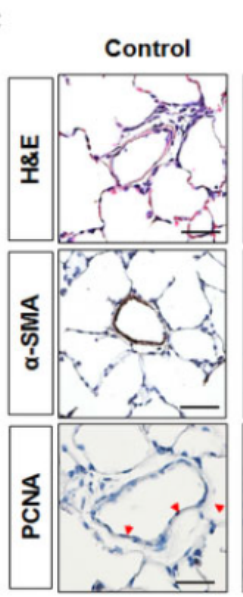

SuHx +
Vehicle
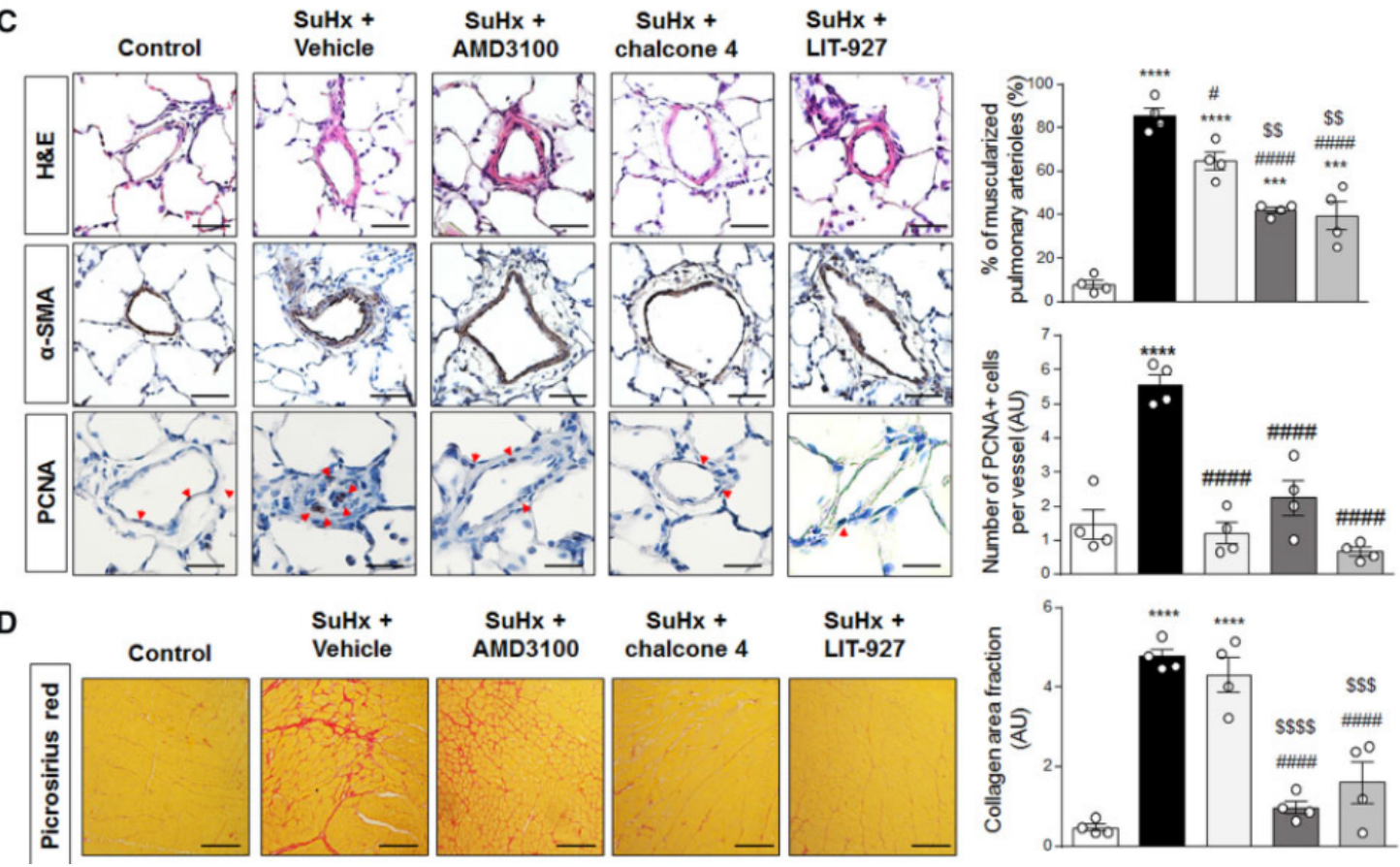

Figure 5: CXCL12 neutralization inhibits the migration potentials of Human PA-SMCs and pulmonary pericytes in vitro. (A) Representative images of wound healing assay and quantification of the surface covered by human PA-SMCs after 0,12 , and 24 hours exposed or not to AMD3100, chalcone 4, or LIT-927 at the indicated doses. (B) Representative images and quantification of the in vitro migration of pulmonary pericytes in the modified Boyden exposed or not to AMD3100, chalcone 4 , or LIT-927 at the indicated doses for 6 hours. Horizontal lines display the mean \pm SEM $(n=4-6)$. Comparisons were made using 1-way ANOVA with Tukey's post hoc tests. * $p$-value $<0.05$; ${ }^{* *} p$-value $<0.01 ;{ }^{* * * *} p$-value $<0.0001$ versus vehicle-treated cells. $\$ p$-value $<0.05 ; \$ \$ p$-value $<0.01 ; \$ \$ \$ p-$ value < 0.001; $\$ \$ \$$-value $<0.0001$ versus AMD3100-treated cells. Scale bar $=500 \mu \mathrm{m}$ in all sections. $A U=$ arbitrary unit. 

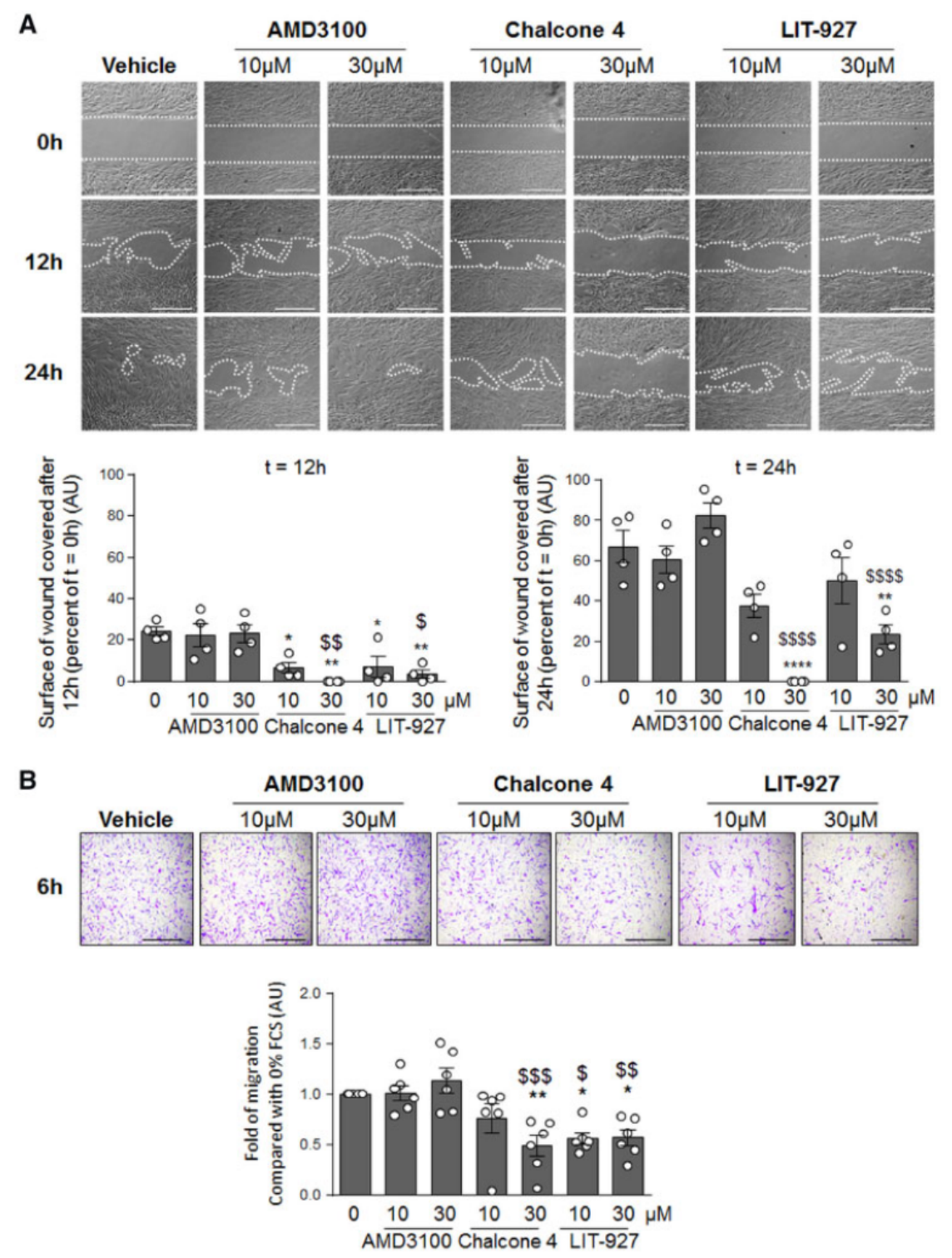

Figure 6: CXCL12 neutralization decreases pericyte coverage of vessels as well as macrophage infiltration in lungs of MCT-injected and SuHx rats. (A) Representative Western blot and quantification of the NG2: $\beta$-actin ratios in lungs from control, MCT-injected and SuHx rats treated or not with vehicle, AMD3100, chalcone 4, or LIT-927. (B) Representative images of 3G5 (white) with SM22 (green) and DAPI (blue) and quantification of the number of 3G5 positive cells per vessels in lungs of MCT-injected and SuHx rats treated or not with vehicle, AMD3100, chalcone 4, or LIT-927. Horizontal lines display the mean \pm SEM $(n=4-5) .{ }^{*} p$-value $<0.05 ;{ }^{* * *} p$-value $<0.001 ;{ }^{* * * *} p$-value $<0.0001$ versus controls. Comparisons were made using 1-way ANOVA with Tukey's post hoc tests. \# p-value < 0.05; \#\# p-value < 0.01; \#\#\# p-value < 0.001; \#\#\# p-value $<0.0001$ versus vehicle treated rats. $\$ \$ p$-value $<0.01 ; \$ \$ \$$-value $<0.001$ versus $A M D 3100$-treated cells. $A U=$ arbitrary unit; DAPI = 4',6-diamidino-2-phenylindole. 
A
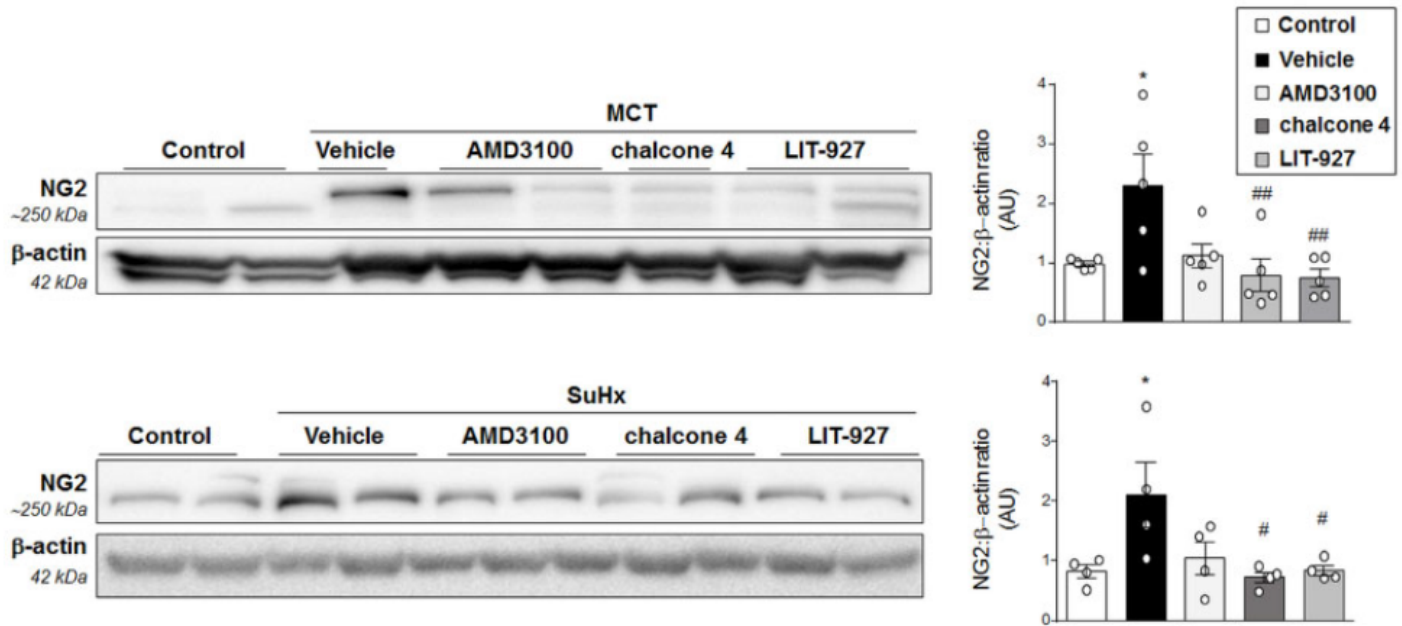

B

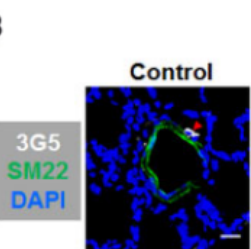

MCT +
Vehicle

MCT +
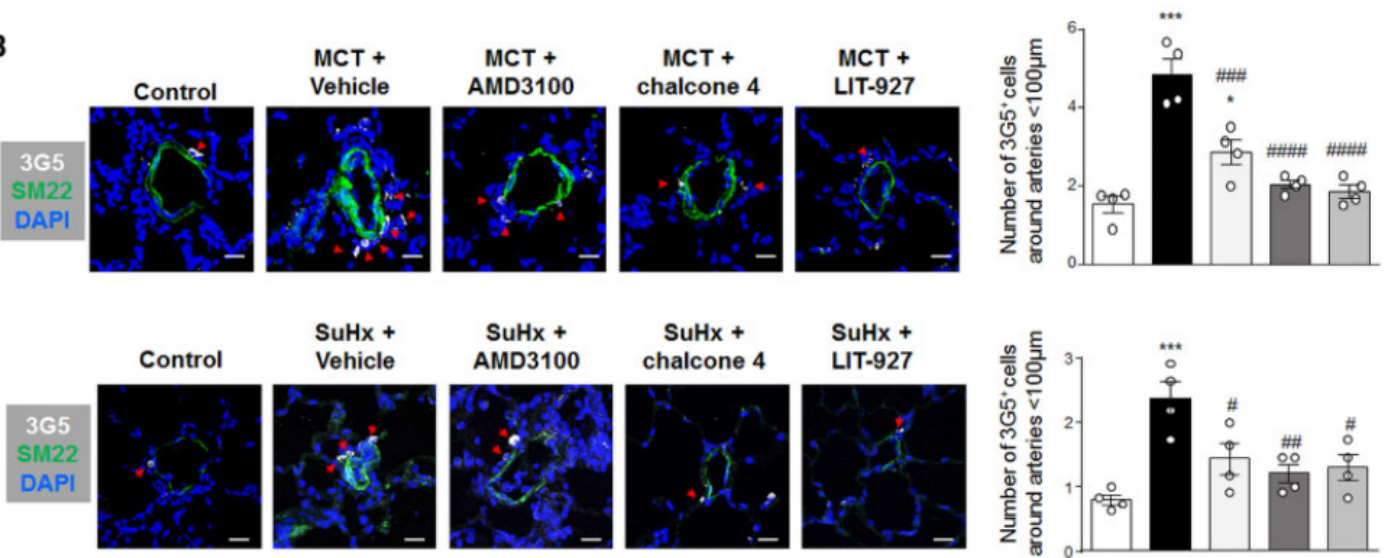

SuHx +

SuHx +

SuHx +

SuHx +
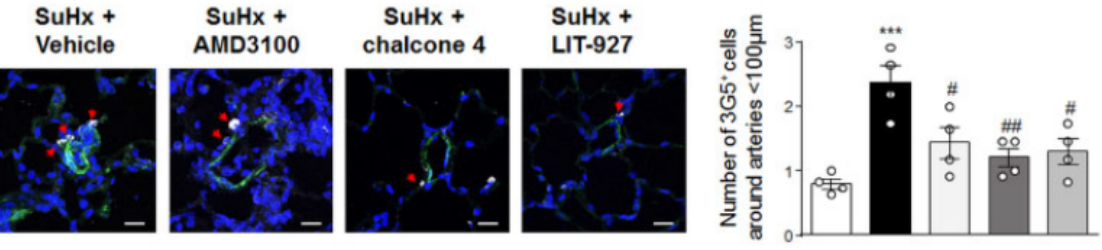

C
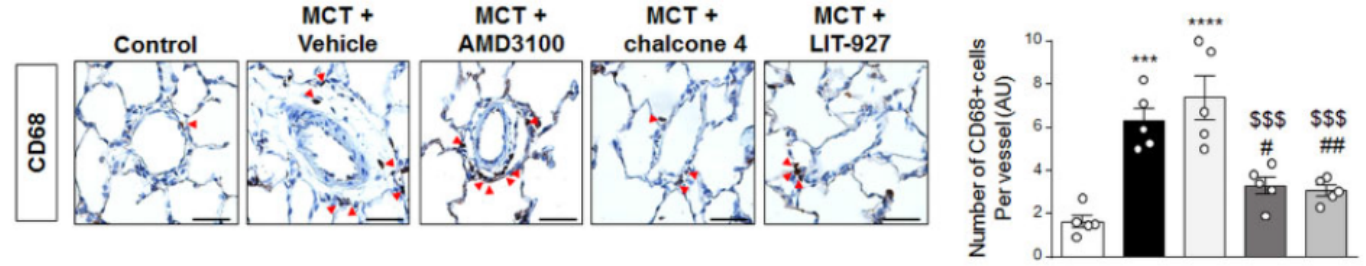

D
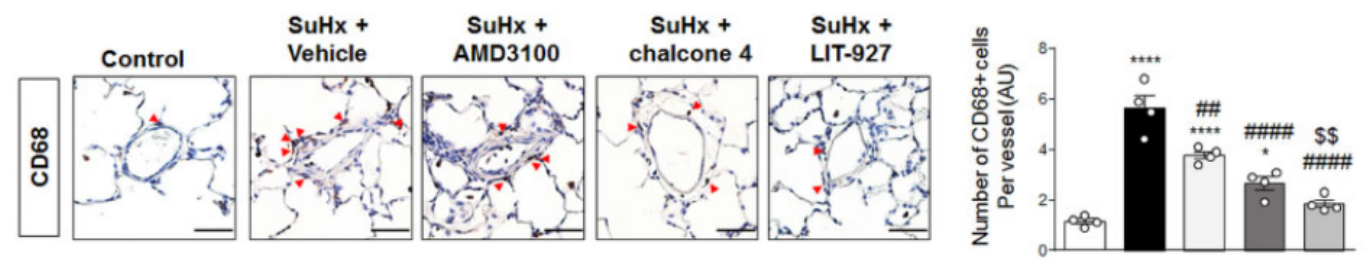